\title{
Transport Mechanism of Guest Methane in Water-Filled Nanopores
}

\author{
Tai Bui, ${ }^{\dagger}$ Anh Phan, ${ }^{\dagger}$ David R. Cole, ${ }^{\ddagger}$ and Alberto Striolo ${ }^{*}{ }^{\dagger}$ (i) \\ ${ }^{\dagger}$ Department of Chemical Engineering, University College London, London WC1E 7JE, U.K. \\ ${ }^{\ddagger}$ School of Earth Sciences, The Ohio State University, Columbus, Ohio 43210, United States
}

\section{Supporting Information}

\begin{abstract}
We computed the transport of methane through $1 \mathrm{~nm}$ wide slit-shaped pores carved out of selected solid substrates using classical molecular dynamics simulations. The transport mechanism was elucidated via the implementation of the well-tempered metadynamics algorithm, which allowed for the quantification and visualization of the free energy landscape sampled by the guest molecule. Models for silica, magnesium oxide, alumina, muscovite, and calcite were used as solid substrates. Slit-shaped pores of width 1 $\mathrm{nm}$ were carved out of these materials and filled with liquid water. Methane was then inserted at low concentration. The results show that the diffusion of methane through the hydrated pores is strongly dependent on the solid substrate. While methane molecules diffuse isotropically along the directions parallel to the pore surfaces in most

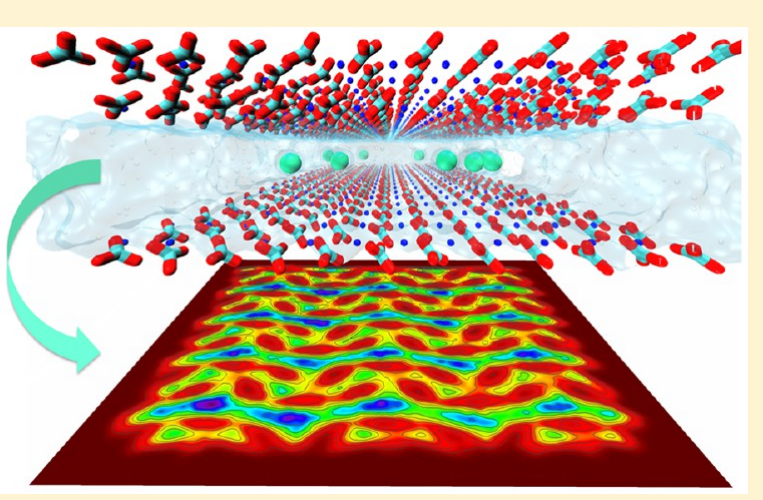
of the pores considered, anisotropic diffusion was observed in the hydrated calcite pore. The differences observed in the various pores are due to local molecular properties of confined water, including molecular structure and solvation free energy. The transport mechanism and the diffusion coefficients are dependent on the free energy barriers encountered by one methane molecule as it migrates from one preferential adsorption site to a neighboring one. It was found that the heterogeneous water distribution in different hydration layers and the low free energy pathways in the plane parallel to the pore surfaces yield the anisotropic diffusion of methane molecules in the hydrated calcite pore. Our observations contribute to an ongoing debate on the relation between local free energy profiles and diffusion coefficients and could have important practical consequences in various applications, ranging from the design of selective membranes for gas separations to the sustainable deployment of shale gas.
\end{abstract}

\section{INTRODUCTION}

Molecular diffusion generally refers to the thermal motion of molecules in space. The diffusivity or diffusion coefficient of a substance is an important parameter representing the mobility of molecules. The diffusion coefficient depends on many factors including the thermodynamic conditions of the system, the physical-chemical properties of the molecules themselves, and their interactions with surrounding molecules. Molecular diffusion plays a crucial role in various industrial processes and technological applications. For example, in gas separation processes using membranes, the difference in the diffusion coefficients through pores or channels is one of the factors governing the membranes' selectivity. In the petroleum industry, hydraulic fracturing is becoming one of the most important processes to produce gas and oil from subsurface formations with extremely low permeability, in which molecular diffusion could be one of the factors determining the productivity of a formation. ${ }^{1}$ It is possible that the imbibition of fracturing fluids into the rock matrix due to strong interactions with substrate surfaces causes fluid loss ${ }^{2,3}$ and perhaps also affects the formation productivity. ${ }^{4}$ Considering another science sector, membrane-based drug delivery devices, characterized by both nanopores and nanochannels, promise the ability to precisely manipulate the diffusion of selected compounds using physical and electrical stimuli. ${ }^{5,6}$
For the applications described above, and many others, understanding the transport behavior of molecules confined inside narrow pores is crucial. Unlike molecules in bulk fluid phases, which usually possess isotropic properties, molecules under confinement can behave differently due to strong interactions with the substrate material, preferential adsorption, and crowding effects due to the presence of other molecules near the interfaces. ${ }^{7,8}$ Thus, properties predicted and measured in bulk phase are not adequate for describing the behavior of molecules confined in nanoporous systems. 9 The diffusion coefficient of molecules in narrow pores is expected to be dimension- and geometry-dependent. For example, most molecular dynamics (MD) simulation studies on fluid diffusion in slit-shaped pore systems confirmed that the diffusion coefficients in the directions parallel and perpendicular to the solid surfaces are different. ${ }^{10,1 \mathrm{f}}$ Franco et al. ${ }^{11}$ recently reported that the diffusion of methane, nitrogen, and carbon dioxide confined inside narrow calcite pores is anisotropic even in the directions parallel to the pore surfaces. The anisotropic behavior is believed to be due to the heterogeneous arrangement of ions in the solid crystals as well as to the

Received: March 22, 2017

Revised: $\quad$ May 4, 2017

Published: May 11, 2017 


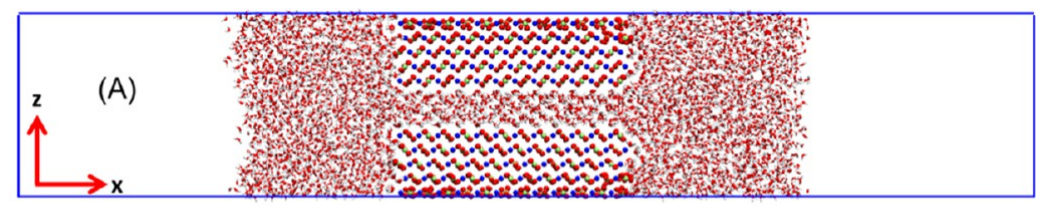

(B)

(C)

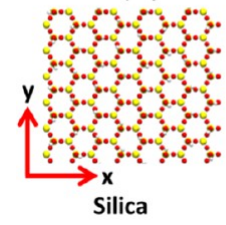

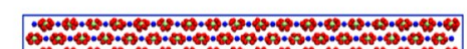

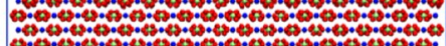

2

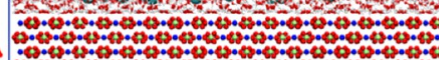
(1).4. $\rightarrow y$

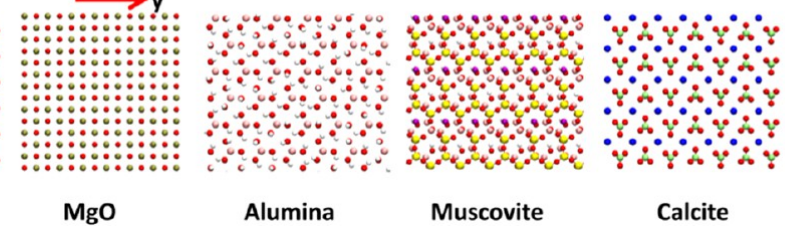

Figure 1. (A) Representative simulation snapshot for the initial configuration of the finite-pore system in which the pore is filled with water. (B) Representative simulation snapshots for the initial configuration of methane confined in the infinite pore saturated with water. (C) Top view of silica, $\mathrm{MgO}$, alumina, muscovite, and calcite substrate surfaces from the left to right, respectively. $\mathrm{Si}=$ yellow; $\mathrm{Mg}=\tan ; \mathrm{Al}=$ pink; $\mathrm{K}=$ purple; $\mathrm{C}=$ green; $\mathrm{Ca}=$ blue; $\mathrm{O}=$ red; $\mathrm{H}=$ white.

strong interactions between diffusing molecules and the calcite surfaces. Recent studies ${ }^{12,13}$ also suggested that the complex diffusion mechanism in confinement is not solely based on the static properties of the systems, such as one-dimensional free energy profiles, but also on its dynamic evolution, such as density fluctuations.

In an attempt to clarify the diffusion behavior of gas molecules (methane) guests in hydrated slit-shaped nanopores, we employ here classic MD simulations. We quantify the selfdiffusion coefficients of the guest methane along the directions parallel to the solid substrate within slit-shaped pores. No chemical potential gradient is applied. The pores considered were obtained from five different substrates (silica, magnesium oxide, alumina, muscovite, and calcite). All pores were filled with liquid water. By analyzing solvation free energy (which is related to the density fluctuations of water), 3-dimensional free energy landscapes, and trajectories of methane molecules, we provide insights into the diffusion mechanism of the guest methane.

The remainder of the article is organized as follows: In the Methods section we first describe force fields and simulation protocols; we then describe the simulation algorithms, including the metadynamics approach, and the methods used to analyze the results. In the Results section we provide a summary of the observations, and we refer to the Supporting Information for additional observations. We conclude with a summary of the main observations, with emphasis on molecular mechanisms.

\section{METHODS}

We conducted a series of equilibrium molecular dynamics (MD) simulations. We describe here, briefly, the models implemented to simulate the solid substrates, the force fields implemented to model water and methane, as well as their interactions with the solids, the setup of the simulated systems, and the algorithms, with particular attention to the implementation of metadynamics.

Solid Supports. We considered slit-shaped pores obtained from five model materials: silica, alumina, $\mathrm{MgO}$, muscovite, and calcite. The systems are shown in Figure 1. Details about silica, alumina, $\mathrm{MgO}$, and muscovite model materials have been provided elsewhere. ${ }^{14-21}$ Calcite slabs were obtained from a calcite crystal terminated at the $\{10 \overline{1} 4\}$ plane. ${ }^{22}$ All the nonbridging oxygen atoms on silica and alumina surfaces were protonated, ${ }^{17,18}$ while the $\mathrm{MgO}$, muscovite, and calcite surfaces were not hydroxylated. ${ }^{23}$ Although these surfaces are oversimplifications, the protonation states are among those expected under hydrated conditions (e.g., Kerisit et al. ${ }^{24}$ and Lardge et al. ${ }^{25}$ reported that no water dissociation is expected on the $\{10 \overline{1} 4\}$ calcite surface). The substrates have a surface parallel to the $X-Y$ plane of the simulation box. The $X$ and $Y$ dimensions of the substrates were of $51.7 \times 100.8,46.9 \times 90.7$, $52.6 \times 105.1,47.2 \times 73.4$, and $48.6 \times 90.0 \AA^{2}$ for silica, alumina, $\mathrm{MgO}$, muscovite, and calcite, respectively. Each pore was obtained by separating the solid substrates along the $Z$ direction, which is perpendicular to the $X-Y$ plane. The $Z$ dimension of the simulation box, which includes the pore and the solid substrate, was set to $42.9,33.8,33.1,46.3$, and $38.9 \AA$ for silica, alumina, $\mathrm{MgO}$, muscovite, and calcite pores, respectively. These dimensions allowed us to maintain the pore width in all the systems constant at $10 \AA$. The pore width was defined as the shortest center-to-center distance between surface oxygen atoms across the pore volume.

Force Fields. As in our prior works, the CLAYFF force field was implemented to simulate silica, alumina, $\mathrm{MgO}$, and muscovite substrates. ${ }^{26}$ Calcite was described using the force field developed by Xiao et al. ${ }^{27}$ For the calcite substrate, the calcium and carbon atoms were kept rigid, whereas the oxygen atoms were allowed to move freely. The silicon, aluminum, and oxygen atoms were held at fixed positions while the surface hydroxyl hydrogen atoms were allowed to vibrate. All atoms in $\mathrm{MgO}$ were kept rigid.

For the case of silica, alumina, $\mathrm{MgO}$, and muscovite pore systems, the transferable potentials for phase equilibria (TraPPE) force field were implemented to model methane, ${ }^{28}$ and the rigid SPC/E model was used to simulate water. ${ }^{29}$ Bond lengths and angles of water were kept fixed by implementing the SETTLE algorithm. ${ }^{30}$ For the system containing calcite, the model developed by Xiao et al. ${ }^{27}$ was implemented to describe calcite-water interactions. To be consistent with the original 
force field, the TIP3P model ${ }^{31}$ was employed to model water in calcite and the OPLS force field ${ }^{32}$ to model methane.

Nonbonded interactions were modeled by means of dispersive and electrostatic forces. The electrostatic interactions were modeled by the Coulombic potential, with long-range corrections treated using the particle mesh Ewald (PME) method. $^{33}$ Dispersive interactions were modeled by $12-6$ Lennard-Jones (LJ) potentials. The LJ parameters for unlike interactions were determined by Lorentz-Berthelot combining rules $^{34,35}$ from the values of like components. The cutoff distance for all interactions was set to $9 \AA$.

Simulation Protocols. In all cases the simulation box was periodic in the three directions. Two configurations of the pore were used in this study: finite and infinite pores.

In finite-pore systems, the simulation boxes were extended on both sides of the pore along the $X$ direction until the $X$ dimension reached 19.2, 19.3, 18.8, 32.4, and $32.8 \mathrm{~nm}$ for silica, $\mathrm{MgO}$, alumina, muscovite, and calcite, respectively. Then, a sufficient amount of water $(10060,8560,8560,8868$, and 9500 water molecules for silica, $\mathrm{MgO}$, alumina, muscovite, and calcite pores, respectively) was used to fill the pore volume and create thin layers on the solid substrate outside of the pores, leaving empty the rest of the simulation box in the $X$ direction. One representative simulation snapshot for these finite-pore systems is shown in Figure 1A. These systems were used to obtain the density of water molecules confined within the various pores. To achieve this, $\mathrm{MD}$ simulations at constant number of molecules $(N)$, constant volume $(V)$, and constant temperature $(T=300 \mathrm{~K})$ were conducted for $40 \mathrm{~ns}$. Equilibrium was considered achieved when both the density profiles of water inside the pores and the total energy of the systems converged. These simulations converged when 1536, 1446, 1086, 900, and 1542 water molecules, respectively, were adsorbed in the pores.

In infinite-pore systems, the $X$ and $Y$ dimensions of the simulation boxes were set to be equal to those of the solid substrates, reflecting the periodicity of the solid crystalline substrates. Thus, because of periodic boundary conditions, the nanopores become effectively infinite along both the $X$ and $Y$ directions. The number of water molecules inserted in the infinite-pore systems was identified from the finite-pore simulations. Ten methane molecules were then inserted randomly inside the hydrated pores. One representative simulation snapshot of the infinite-pore system is shown in Figure 1B. To equilibrate these systems, one NVT equilibrium simulation was conducted for $20 \mathrm{~ns}$. The convergence of water and methane density profiles along the $Z$ direction of the simulation box was evaluated to ensure equilibration was reached. The data were then collected during 25 ns NVT production runs at $300 \mathrm{~K}$, after the $20 \mathrm{~ns}$ equilibration run (total simulation time was of $45 \mathrm{~ns}$ per system).

Algorithms. $\mathrm{MD}$ simulations were conducted using the package GROMACS, ${ }^{36,37}$ version 5.1.2. The simulated temperature was maintained at $300 \mathrm{~K}$ by a Nosé-Hoover thermo$\mathrm{stat}^{38,39}$ with a relaxation time of $500 \mathrm{fs}$. The equations of motion were solved by implementing the leapfrog algorithm ${ }^{40}$ with 1.0 fs time steps.

Metadynamics. Well-tempered metadynamics simulations ${ }^{41}$ were conducted to quantify the three-dimensional free energy landscape experienced by a guest methane molecule confined within the hydrated pores. Metadynamics is an enhanced sampling algorithm in which the free energy landscape is explored by introducing a bias potential on one or more collective variables (CVs). The bias potential is dynamically deposited to the potential energy landscape of the system along the trajectory in the CVs space. As a consequence, the system is forced to explore configurations at higher and higher potential energy as the bias is systematically added. Details on the algorithm and its recent developments can be found elsewhere. ${ }^{41,42}$ The convergence of the well-tempered metadynamics approach depends on the judicious choice of appropriate sets of CVs, the height and width of the Gaussians used to add the bias potential, and the "bias factor". The CVs chosen for the present study are the components of the distance along the three Cartesian coordinates $(X, Y, Z)$ between one methane molecule and the center of the pore. To limit the space explored, we applied two soft walls at $-1 \mathrm{~nm}$ (lower wall) and $1 \mathrm{~nm}$ (upper wall) for each CV (i.e., the free energy landscape was explored for one methane molecule within a cube of size $2 \mathrm{~nm}$ centered in the center of the pore; note that the bias potential was not large enough to force the methane against the atoms of the solid substrate). The Gaussian height was set at $0.5 \mathrm{~kJ} / \mathrm{mol}$, and the bias factors were set to 20. The Gaussian width was set at 0.02 for the CVs along the $X$ and $Y$ directions and 0.01 for that along the $Z$ direction. These values were set equal to or less than $1 / 5$ of the standard deviation of the CVs observed during unbiased equilibrium MD simulations. In the methadynamics simulations only one methane molecule was confined within the infinite hydrated pores, and 1536 and 1542 water molecules were present within the silica and calcite pores, respectively.

All well-tempered metadynamics simulations were conducted at $300 \mathrm{~K}$ in a canonical ensemble (NVT) using the GROMACS package $^{36}$ version 5.1.2 integrated with the PLUMED package $^{42}$ version $2.3 \mathrm{~b}$. For these simulations, we employed the Nosé-Hoover thermostat with a relaxation time of $500 \mathrm{fs}$. Simulations ran for at least $0.5 \mu$ s until the free energy profiles converged.

Data Analysis. Self-diffusion calculations were based on the mean-square displacement (MSD) approach along the $X$ and $Y$ dimensions separately or within the $X-Y$ plane. The corresponding self-diffusion coefficients $D_{X}$ or $D_{Y}$ or $D_{X Y}$ were calculated using the Einstein relation: ${ }^{43,44}$

$$
\begin{aligned}
& D_{X}=\frac{1}{2} \lim _{t \rightarrow \infty} \frac{\left\langle\left|r_{X i}(t)-r_{X i}(0)\right|^{2}\right\rangle}{t} \\
& D_{Y}=\frac{1}{2} \lim _{t \rightarrow \infty} \frac{\left\langle\left|r_{Y i}(t)-r_{Y i}(0)\right|^{2}\right\rangle}{t} \\
& D_{X Y}=\frac{1}{4} \lim _{t \rightarrow \infty} \frac{\left\langle\left|r_{X Y i}(t)-r_{X Y i}(0)\right|^{2}\right\rangle}{t}
\end{aligned}
$$

In eqs 1,2 , and $3 r_{X i}(t), r_{Y i}(t)$, and $r_{X Y i}(t)$ are the positions along the $X$ and $Y$ directions and within the $X-Y$ plane of the simulation box, respectively, of molecule $i$ at time $t$. In these equations angular brackets denote ensemble averages.

To obtain the solvation free energy, we implement the method described in our previous study, ${ }^{12}$ which is based on the quantification of time-averaged water density fluctuations. These are calculated via the probability of observing $N$ molecules within a $6.6 \AA$ diameter spherical volume, $v$, located at the center of the hydrated pores: ${ }^{45}$

$$
P_{v}(N)=\left\langle\delta\left(N_{v}-N\right)\right\rangle=\lim _{t \rightarrow \infty}\left[\frac{1}{t} \int_{0}^{t} \mathrm{~d} t^{\prime} \delta\left(N_{v}\left(t^{\prime}\right)-N\right)\right]
$$



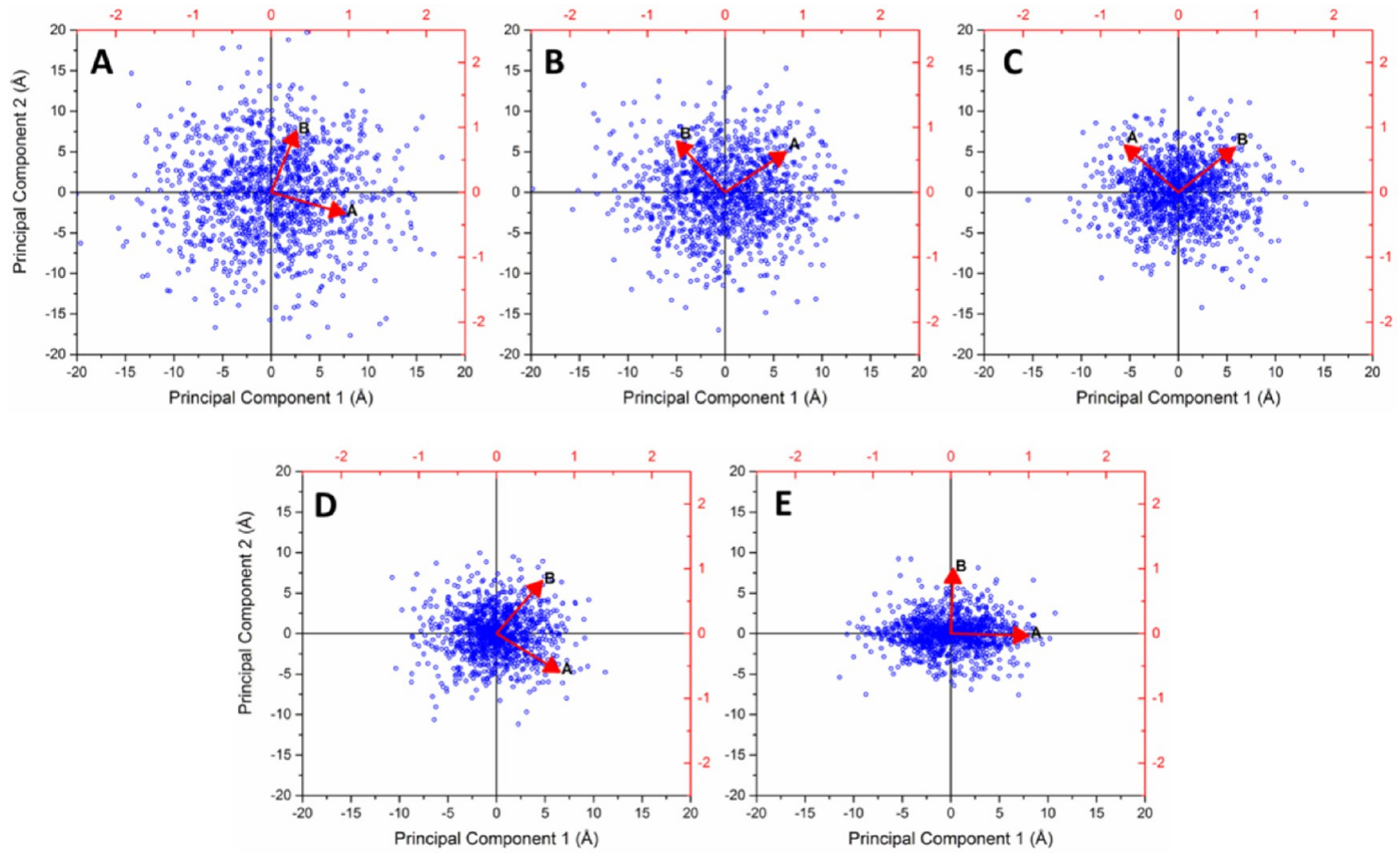

Figure 2. End point of the vectors connecting the positions of methane molecules at time $t$ and $t+\Delta t(\Delta t=200 \mathrm{ps})$ during $25 \mathrm{~ns}$ NVT simulations in silica (A), $\mathrm{MgO}$ (B), alumina (C), muscovite (D), and calcite (E) pores. The data were used to conduct a principal component analysis. The red vectors represent the eigenvectors, which we use to identify the preferential diffusion directions of methane in the $X-Y$ plane of the pores. When the two eigenvalues in a pore are similar, the diffusion is isotropic. When they are different, as in the case of calcite pores (panel E), the diffusion is anisotropic.

Table 1. Principal Component Analysis of the Vectors Describing the Migration of Methane Guest Molecules in 200 ps in Different Pores

\begin{tabular}{|c|c|c|c|c|c|c|}
\hline & \multirow[b]{2}{*}{ eigenvalue 1} & \multirow[b]{2}{*}{ eigenvalue 2} & \multicolumn{2}{|c|}{ eigenvector 1} & \multicolumn{2}{|c|}{ eigenvector 2} \\
\hline & & & $X$ & $Y$ & $X$ & $Y$ \\
\hline silica & 32.8 & 31.6 & 0.946 & -0.324 & 0.324 & 0.946 \\
\hline $\mathrm{MgO}$ & 22.9 & 21.5 & 0.783 & 0.621 & -0.621 & 0.783 \\
\hline alumina & 14.1 & 12.1 & -0.690 & 0.723 & 0.723 & 0.690 \\
\hline muscovite & 9.0 & 7.9 & 0.810 & -0.587 & 0.587 & 0.810 \\
\hline calcite & 11.1 & 4.0 & 0.999 & -0.03 & 0.03 & 0.999 \\
\hline
\end{tabular}

Then the solvation free energy is calculated through the relation $^{46}$

$$
\Delta \mu_{v}=-k_{\mathrm{B}} T \ln P_{v}(0)
$$

In eq $5, k_{\mathrm{B}}$ is Boltzmann's constant and $T$ is temperature.

Two-dimensional free energy maps in metadynamics simulations were obtained by integrating the bias potential deposited during the simulation with respect to two collective variables at a time $(X-Z, Y-Z$, and $X-Y)$ using the utility sum_hills implemented in the PLUMED package.

Representative trajectories of methane molecules were collected during $25 \mathrm{~ns}$ of production simulation runs and then projected in the $X-Z, Y-Z$, and the $X-Y$ planes.

\section{RESULTS AND DISCUSSION}

Self-Diffusion of Methane. Simulations were conducted to quantify the self-diffusion coefficients of the guest methane molecules within the different hydrated pores. The twodimensional self-diffusion coefficient within the $X-Y$ plane (parallel to the pore surfaces) was estimated from mean-square displacement data (data in Figure S1 of the Supporting Information). To quantify whether the diffusion is isotropic or not, we extracted a data set of two-dimensional vectors (two components: $X$ and $Y$ ) connecting the positions of methane molecules at time $t$ and $t+\Delta t$ (with $\Delta t=200 \mathrm{ps).} \mathrm{The} \mathrm{data}$ were collected during $25 \mathrm{~ns}$ of equilibrated simulations. The end-points of these vectors are shown in Figure 2. The preferential directions for the diffusion of methane molecules in the pores can be identified by applying the principal component analysis (PCA) method ${ }^{47}$ on these data sets. The concept is to determine the principal components that maximize the variance of the data and that simultaneously minimize the reconstruction errors. Such preferential directions were then identified from eigenvectors and eigenvalues from the tabulated data sets. 
The results are shown in Table 1 . These results indicate the following: when the eigenvalues in a pore are similar, the diffusion is isotropic within the $X-Y$ plane; when the eigenvalues are different (as in the case of the calcite pore), the diffusion is anisotropic. In Figure 2 we show the eigenvectors in each pore as red arrows. When the diffusion is isotropic, the $X$ and $Y$ components of the self-diffusion coefficient are expected to be similar; when the diffusion is anisotropic, the $X$ and $Y$ components are expected to be different. In what follows these differences are quantified.

Figure 3 provides a comparison of the $2 \mathrm{D}$ self-diffusion coefficients (blue bars) simulated for methane in water

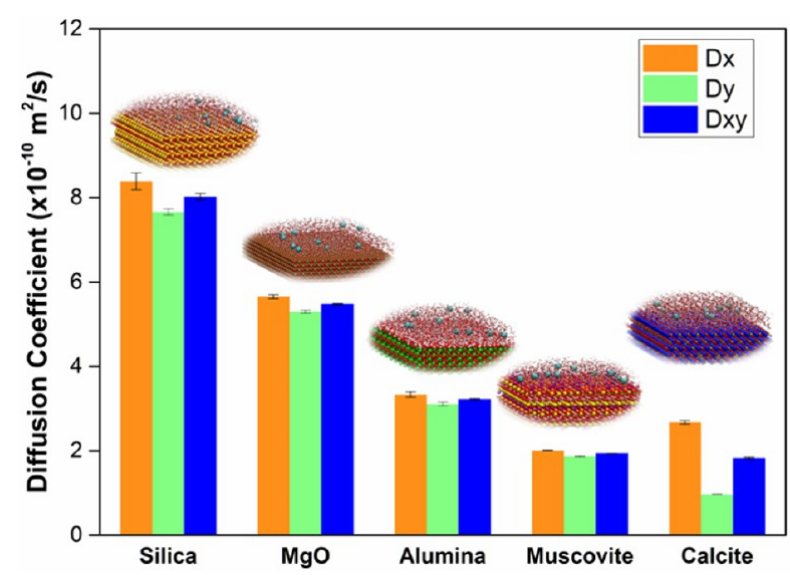

Figure 3. Self-diffusion coefficients of methane molecules within five different pores saturated with water. For each type of pore, the left, middle, and right columns represent the self-diffusion coefficient in the $X$ and $Y$ directions and the $2 \mathrm{D}$ self-diffusion coefficient within the $X-Y$ plane, respectively.

confined in the five pores. The results show that the diffusion of methane in the water-saturated narrow pores strongly depends on the solid substrates. Note that in all cases the water density near the center of the pore is $\sim 1 \mathrm{~g} / \mathrm{cm}^{3}$ (see Figure S3). The 2D self-diffusion coefficient of methane was found to vary in the following order: silica pore $>\mathrm{MgO}>$ alumina $>$ muscovite $\cong$ calcite pore. For comparison, the self-diffusion coefficient of methane in bulk liquid water is found to be $\sim 19 \times$ $10^{-10} \mathrm{~m}^{2} / \mathrm{s}$ experimentally at $T=298 \mathrm{~K}^{48}$ or $\sim 20 \times 10^{-10} \mathrm{~m}^{2} / \mathrm{s}$ by MD simulations at $T=300 \mathrm{~K},{ }^{49}$ which is higher than that obtained in all the hydrated pores studied here.

In Figure 3, we also report the self-diffusion coefficients calculated for the guest methane molecules within the five pores along the $X$ and $Y$ directions (orange and green bars, respectively). For the hydrated silica, $\mathrm{MgO}$, alumina, and muscovite pores the differences between data obtained along the $X$ and $Y$ directions are minimal, confirming that the diffusion of methane in these hydrated pores is isotropic. The results for the hydrated calcite pore, however, show that the methane self-diffusion coefficient along the $X$ direction is noticeably larger (almost by a factor of 3 ) than that obtained along the $Y$ direction. This unexpected result, consistent with the PCA analysis of Figure 2, shows that the diffusion of methane is highly anisotropic in this system.

The remainder of the article aims at understanding the underlying molecular mechanisms responsible for the differences observed between the calcite compared to the other pore types.
Self-Diffusion of Water. Because our prior work suggests that the water structure and the network of water-water hydrogen bonds are responsible for the transport properties of methane molecules confined in hydrated pores, ${ }^{12}$ the results shown in Figures 2 and 3 suggest that perhaps water confined in a $1 \mathrm{~nm}$ pore carved out of calcite might also exhibit anisotropic properties, including structure and dynamics. The density profiles for water inside the various pores were calculated as well as the self-diffusion coefficients for those water molecules found in the middle region of the pores, excluding the first and the second layers of water with respect to the pore surfaces. The components of the self-diffusion coefficient were differentiated, similarly to what was done for methane in Figure 3. The results in Figure 4 show that the self-

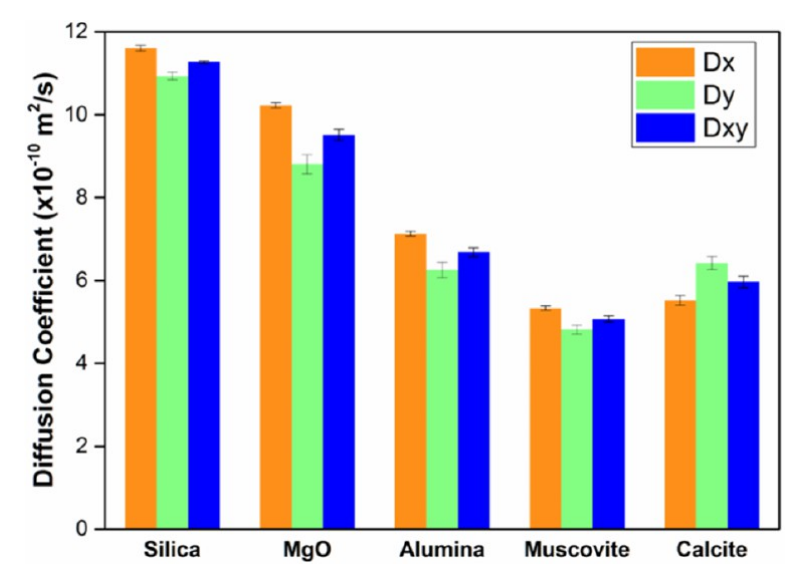

Figure 4. Self-diffusion coefficients calculated for water molecules found within the middle region of five slit-shaped pores saturated with water. For each pore, the left, middle, and right columns represent the self-diffusion coefficients obtained along the $X$ and $Y$ directions and those obtained on the $X-Y$ plane, respectively.

diffusion coefficients of water are substrate-dependent, reflecting in general the order observed for methane selfdiffusion coefficients in the hydrated pores. The main difference is that the self-diffusion coefficient calculated in the calcite pore is larger, rather than approximately the same, than that obtained in muscovite. The differences are, however, not large when compared to the statistical uncertainties.

The self-diffusion coefficients obtained for water are somewhat larger compared to those obtained for methane (compare data in Figure 4 to the correspondent ones in Figure 3). In all cases the self-diffusion coefficient is much lower than that in the bulk liquid water as obtained from experimental data $\left(\sim 24 \times 10^{-10} \mathrm{~m}^{2} / \mathrm{s}\right)^{50,51}$ or from MD simulations with SPC/E model at $298 \mathrm{~K}\left(\sim 28 \times 10^{-10} \mathrm{~m}^{2} / \mathrm{s}\right){ }^{52}$ The data shown in Figure 4 are consistent with results from other studies for confined water. ${ }^{53-56}$

Free Energy Profiles. It is expected that the molecular diffusion of methane in the hydrated pores is primarily governed by the molecular structure of the surrounding environment and by the interactions between the methane molecules and the solid substrate. Comparing the data in Figure 4 to those in Figure 3 suggests that the differences in methane diffusion cannot only be due to differences in water diffusion, although the latter is certainly an important contributor. In an attempt to better understand why the diffusion coefficients of methane molecules reduce monotonically from $\sim 8 \times 10^{-10} \mathrm{~m}^{2} /$ $\mathrm{s}$ in the silica to $\sim 2 \times 10^{-10} \mathrm{~m}^{2} / \mathrm{s}$ in the muscovite and calcite 
pores, the umbrella sampling algorithm was implemented to estimate the height of free energy barriers for one methane molecule moving across the hydrated pores. The methane molecule was forced to remain at a given $X$ position inside the hydrated pore using harmonic springs of elastic constant 3000 $\mathrm{kJ} /\left(\mathrm{mol} \mathrm{nm}^{2}\right)$, and the free energy profile was calculated as the methane molecule moved along the $X$ direction. Details on the umbrella sampling algorithm are described elsewhere. ${ }^{57}$ The results, presented in detail in Figure S5, demonstrate a number of minima, separated by maxima in the free energy profile. The average height of free energy barriers that separate two consecutive minima was calculated, and the results are reported in Figure 5. Note that these results were obtained for methane

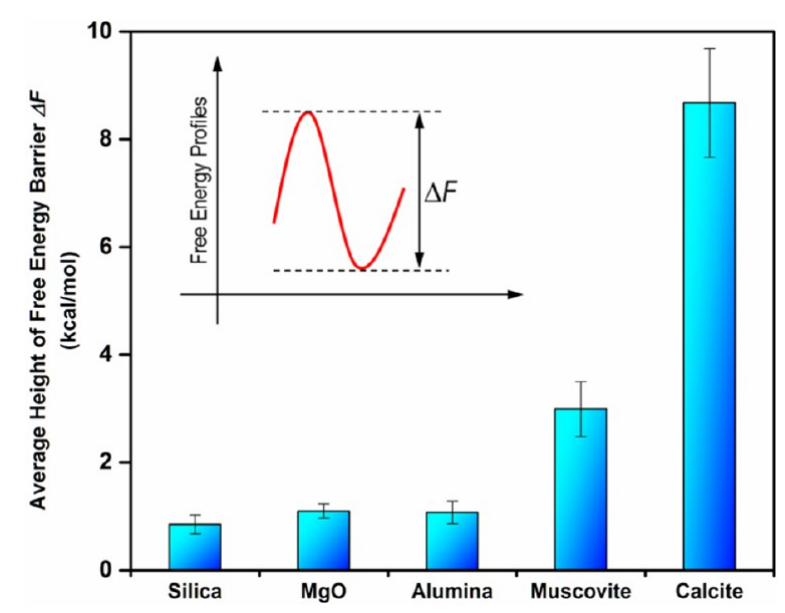

Figure 5. Average height of free energy barrier experienced by one methane molecule moving across hydrated silica, $\mathrm{MgO}$, alumina, muscovite, and calcite pores.

traveling along the $X$ direction. For completeness, these calculations should be repeated at least along the $Y$ direction. Instead, we provide a more detailed analysis in 3D below, using metadynamics.

In general, the results show that as the average height of the free energy barrier increases, the methane self-diffusion coefficient decreases. However, the average height of the free energy barrier is not the only factor governing methane diffusion. In fact, it is likely that the diffusion of water influences that of methane as discussed above. The combined effect of diffusion of water and average height of the barrier separating two neighboring preferential adsorption sites for methane could explain why, for example, while the heights of free energy barriers encountered by methane in the hydrated $\mathrm{MgO}$ and alumina pores are almost identical, the self-diffusion coefficient for methane in the hydrated $\mathrm{MgO}$ pore is larger. Other factors are certainly important, including solvation, hydration structure, free energy landscape, etc., within the hydrated pores considered here. The combination of these factors is expected to yield trajectories reminiscent of random walks.

Solvation Behavior. Our recent work ${ }^{12}$ suggests the possibility that averaged information such as the free energy profiles given in Figure 5 is not sufficient to describe the molecular phenomena attendant with the transport of methane across the hydrated pores. It is possible that methane molecules encounter a dynamically evolving environment, rather than a static one, when adsorbed within the hydrated pores. To quantify this, Limmer et al. ${ }^{45}$ proposed an algorithm to quantify the solvation free energy (SFE) in water as a time-dependent property. In Figure 6, the results for the solvation free energy are presented as a function of position within a $40 \times 40 \AA^{2}$ section of the $X-Y$ plane of the simulation box. Water molecules within a $6.6 \AA$ diameter spherical volume at the center of the hydrated pores were considered for the calculations, and the results were averaged over $10 \mathrm{~ns}$ of simulation. The average solvation free energy is greater for calcite $\cong$ muscovite $>$ alumina $>\mathrm{MgO}>$ silica pore, which corresponds to the decrease of methane diffusion coefficients. These results are consistent with the observation that enhanced density fluctuations (corresponding to low solvation free energy) lead to faster solvent and solute translational dynamics. ${ }^{58}$ It is worth noting that the solvation free energy in this approach is quantified via water density fluctuations. The stronger water fluctuates, the lower the solvation free energy. In our interpretation, water density fluctuations can promote irregular diffusive trajectories for methane through the hydrated pores. We previously showed that when confined in narrow hydrated silica, $\mathrm{MgO}$, and alumina pores, methane molecules travel longer distances when they enter low-density SFE regions, while they remain for longer times near high-density SFE locations. ${ }^{12}$ Such analysis was not repeated in the present paper.

Hydration Structure. To quantify the effect of the molecular hydration structure on the diffusion of methane along the $X-Y$ plane within the hydrated pores, we calculated the in-plane density distributions of water oxygen atoms. In Figure 7, we present the in-plane density distributions of water oxygen atoms as a function of position within a $40 \times 40 \AA^{2}$ section of the $X-Y$ plane. A $1.8 \AA$ thick slab of water located at the center of the hydrated pores was considered. Although results for water density profiles in the direction perpendicular to the pore surfaces (shown in Figure S3) suggest that at the center of the pores water molecules could display a uniform distribution, several literature results show that uniform planar distributions are achieved at distances larger than $\sim 1 \mathrm{~nm}$ from ordered solid substrates such as those considered here. ${ }^{16,17,55,59}$ The results in Figure 7 show preferential distributions for water molecules within each of the pores considered here. The highdensity areas (green-yellow regions) displayed indicate the positions where the water oxygen atoms preferentially reside. The distribution of these areas where water molecules accumulate is periodic on the $X-Y$ plane in all hydrated pores.

Our previous work ${ }^{16,60,61}$ indicated that the distribution of water in the second hydration is due to preferential interactions (i.e., hydrogen bonds) between water molecules in the first and second hydration layers, steric effects, and preferential interactions between water molecules within the second hydration layer. While similar effects are true for the systems considered here, the water distribution within the hydrated calcite pore shown in Figure 7 does not seem to be sufficient to explain why the self-diffusion coefficients of methane along the $X$ and $Y$ directions differ almost by a factor of 3 .

The simulation results indicate self-diffusion coefficients for methane decrease in order from silica, $\mathrm{MgO}$, alumina, muscovite to calcite pores. However, the question regarding what molecular mechanism is responsible for the anisotropic diffusion of methane in the hydrated calcite pore remains unanswered. To test the hypothesis that the hydrogen-bond network formed by water molecules could affect the transport of guest molecules as reported in our previous study, ${ }^{12}$ we calculated the in-plane density distributions of water-water hydrogen bonds (HBs) within the five hydrated pores (Figure 

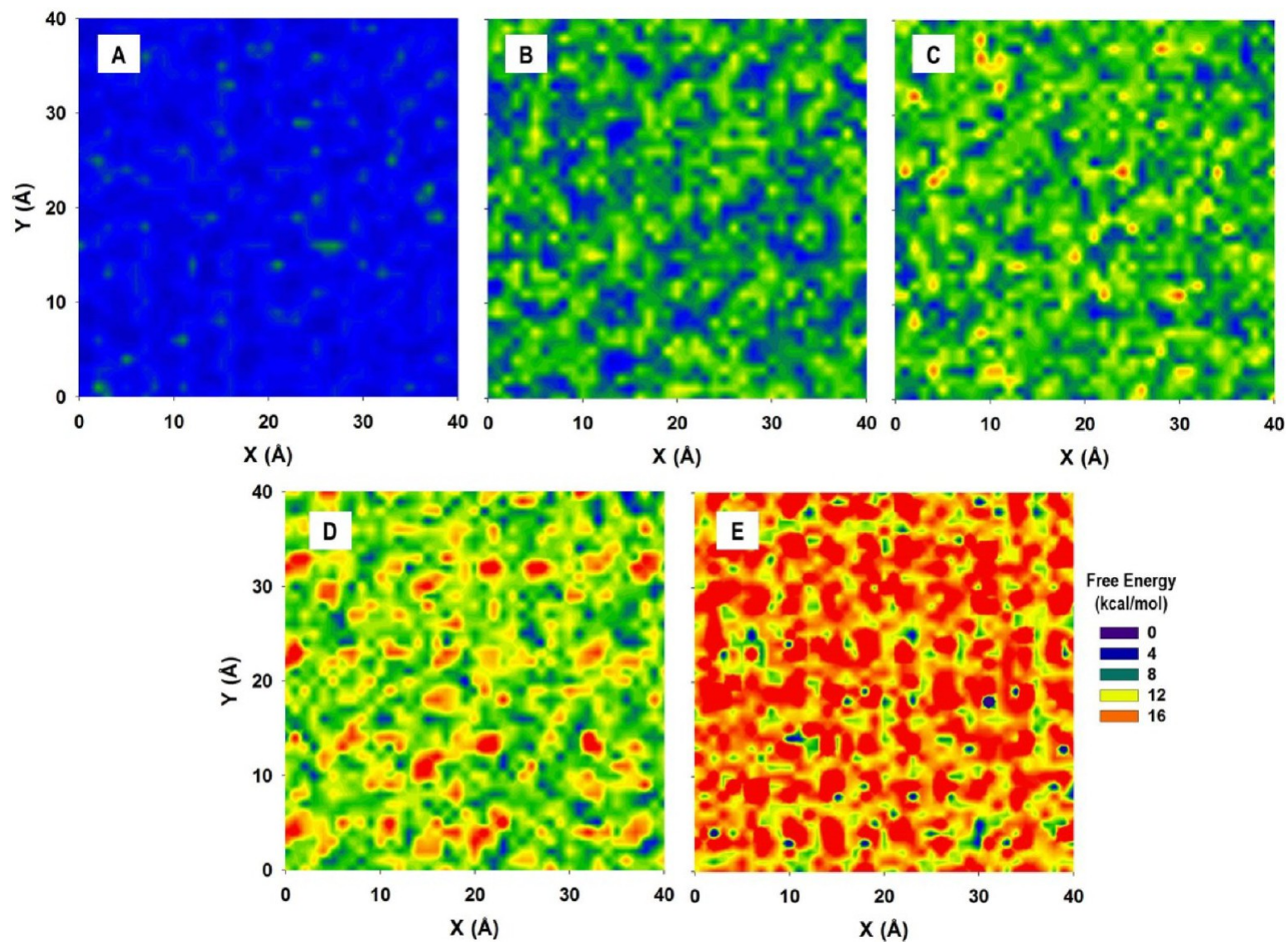

Figure 6. In-plane solvation free energy distributions obtained for the hydrated silica (A), $\mathrm{MgO}(\mathrm{B})$, alumina (C), muscovite (D), and calcite (E) pores. The results were obtained from density fluctuations of water within a $6.6 \AA$ spherical volume located in middle of the pore along $X-Y$ plane and averaged during $10 \mathrm{~ns}$ observations.

8). A somewhat uniform distribution of $\mathrm{HBs}$ is found along both $X$ and $Y$ directions within silica, $\mathrm{MgO}$, alumina, and muscovite hydrated pores. This is not the case within the calcite pore. Within this pore (panel E) a continuous zigzag pattern of maxima and minima of HB density distributed along the $X$ direction is observed, while the distribution along the $Y$ direction is discontinuous. Within the hypothesis that strong water-water $\mathrm{HB}$ networks can provide barriers for the methane diffusion, from the results in Figure 8 it is expected that methane molecules could more easily diffuse along the $X$ direction within the hydrated calcite pore, following the direction of low $\mathrm{HB}$ density paths. Conversely, they would encounter strong barriers when attempting to diffuse along the $Y$ direction, as they would have to overcome high-energy barriers due to the network of water-water HBs. However, by overlaying the density distribution of $\mathrm{HBs}$ to the density distribution of methane (Figure S7), it is observed that methane molecules are more likely to be found along the high $\mathrm{HB}$ density regions. When the density distribution of water molecules in the second hydration layer was compared to the density distribution of methane molecules in the middle of the pore (Figure S8), it was found that methane molecules accumulate in correspondence of low water density regions (note that the low water density is in the second hydration layer, while the methane molecules are in the pore center). In this configuration, methane molecules yield a structure similar to that observed in bulk liquid water (see Figure S9). ${ }^{62-64}$ It was also found that water molecules in the second hydration layer remain in this layer for extensive periods of time (see
Figure S10). The distribution of water molecules in the second hydration layer shows continuous zigzag patterns along the $X$ direction, whereas along the $Y$ direction the maxima are followed by minima as shown in Figure S11. These observations suggest that methane molecules can diffuse more easily along the $X$ direction by following the low-density paths of water molecules in the second hydration layer. This could explain why the self-diffusion of methane in the hydrated calcite pore is $\sim 2.6$ times larger along the $X$ than along the $Y$ direction (see Figure 3).

Free Energy Landscape and Methane Trajectories. Previous free energy calculations using the umbrella sampling technique demonstrate that the methane transport in the narrow pores strongly depends on the surface chemistry of the pores. These calculations were performed only along the reaction coordinate $(\mathrm{RC})$ defined by the $X$ direction of the simulation box. However, the local environment on the plane parallel to the solid substrates within the hydrated pore is more complex; therefore, the potential for mean force (PMF) calculated in a straight RC may not cover the realistic trajectory of methane transport. ${ }^{12}$ In addition, the heterogeneous distribution of water, particularly within the calcite pore, reinforces the possibility that preferential transport pathways for methane exist within hydrated nanopores that follow complex trajectories. Well-tempered metadynamics simulations ${ }^{41}$ were conducted to quantify the free energy landscape in three $X, Y$, and $Z$ directions. We report the results of free energy landscape for two representative pore orientations in silica and calcite. The projections of free energy landscapes on 

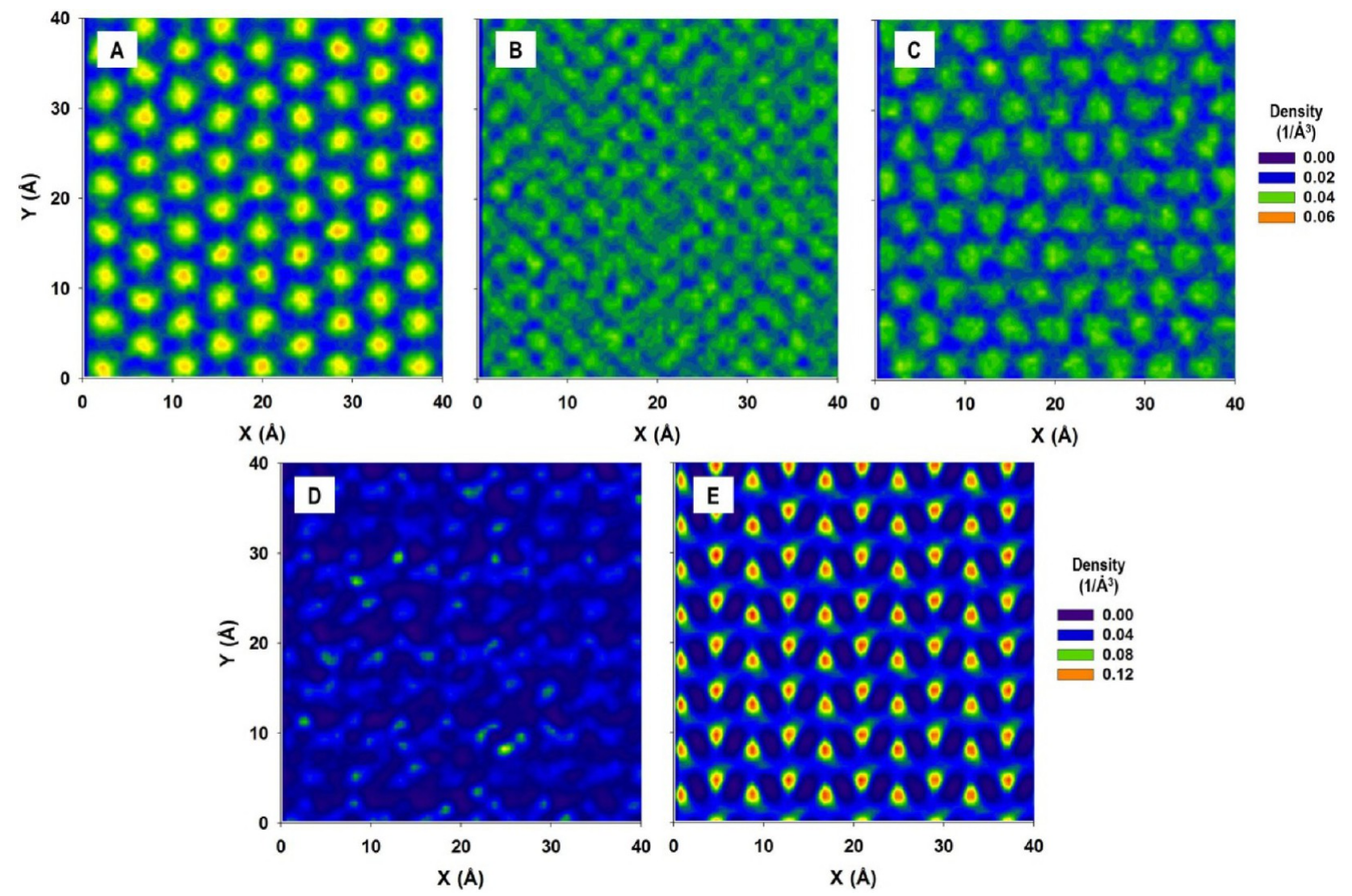

Figure 7. In-plane density distributions of water oxygen atoms found in the middle layer parallel to the $X-Y$ plane within the hydrated silica (A), $\mathrm{MgO}(\mathrm{B})$, alumina (C), muscovite (D), and calcite (E) pores.

the $X-Z, Y-Z$, and $X-Y$ planes are shown in Figure 9 (left, middle, and right panels, respectively). Data obtained for the hydrated silica pore are shown on the top panels; those obtained for the hydrated calcite pore are given in the bottom panels.

Based on the $X-Z$ and $Y-Z$ free energy maps for the hydrated silica pore (top left and middle panels of Figure 9, respectively), the methane molecule can readily diffuse along the $X$ and $Y$ directions in the middle of the pore while encountering rather small energy barriers. The low free energy region is widely distributed in the middle of the pore along the $Z$ direction, which is consistent with the wide peak in the density profile of methane found in that region (see Figure S4). As a consequence of the similarity of the free energy landscapes in the middle of the pore in the $X$ and $Y$ directions, the selfdiffusion coefficient of methane is isotropic in this pore. This is further confirmed by analysis of the $X-Y$ free energy plot (right panel), which shows that the energy wells are distributed uniformly on the surface. These data are consistent with (a) the results shown in Figure 3, (b) the PCA analysis of Figure 2, (c) the distribution of water molecules in different hydration layers of the pore (Figure 7 and Figure S11), and (d) that of methane molecules (Figure S12).

The free energy maps as experienced by methane in the hydrated calcite pore are rather different. These are shown on the bottom panels of Figure 9. The $X-Y$ (panel D) and $X-Z$ (panel E) free energy maps show deep free energy wells located at the center of the pore together with shallow wells located 1.5 $\AA$ far from the center of the pore in the $Z$ direction. These wells are consistent with the methane density profile calculated along the $Z$ direction (see Figure S4) and correspond to the preferential sites within which methane molecules prefer to accumulate at the center of the pore. In Figure 9, we highlight the path of minimum resistance that methane molecules could follow in diffusing along the $X$ (panel $\mathrm{D}$ ) and $Y$ directions (panel E) within the pore. We can observe that the former are straight lines, much less tortuous than the latter, which are zigzags. The $X-Z$ free energy map (panel $D$ ) confirms that the favorable pathway for the methane transport in the $X$ direction is the one connecting free energy wells at the center of the pore. In contrast, the preferable pathway for the methane transport in the $Y$ direction is the one that connects the wells in the middle pore with the wells near the pore surfaces rather than the straight path observed in the $X$ direction. This suggests that the methane molecule can only jump to another position along the $Y$ direction by moving closer to the pore surfaces, where adsorption is less favorable, and then moving back to the center of the pore. Moreover, by comparing the height of free energy barriers encountered as the molecule moves from one energy well to another along the $X$ and $Y$ directions, from Figure 9 (panels D and E) lower barriers are observed as the molecule travels along the $X$ direction $(\sim 5 \mathrm{~kJ} / \mathrm{mol})$ compared to those encountered as the molecule moves along the $Y$ direction $(\sim 10 \mathrm{~kJ} / \mathrm{mol})$. The preferential pathways and the height of free energy barriers seem to explain why the diffusion coefficient of methane molecules calculated in the $X$ direction is greater than that found in the $Y$ direction. Comparing the $X-Y$ free energy maps against the density distributions of methane in the $X-Y$ (Figure S13) suggests that methane molecules diffuse 

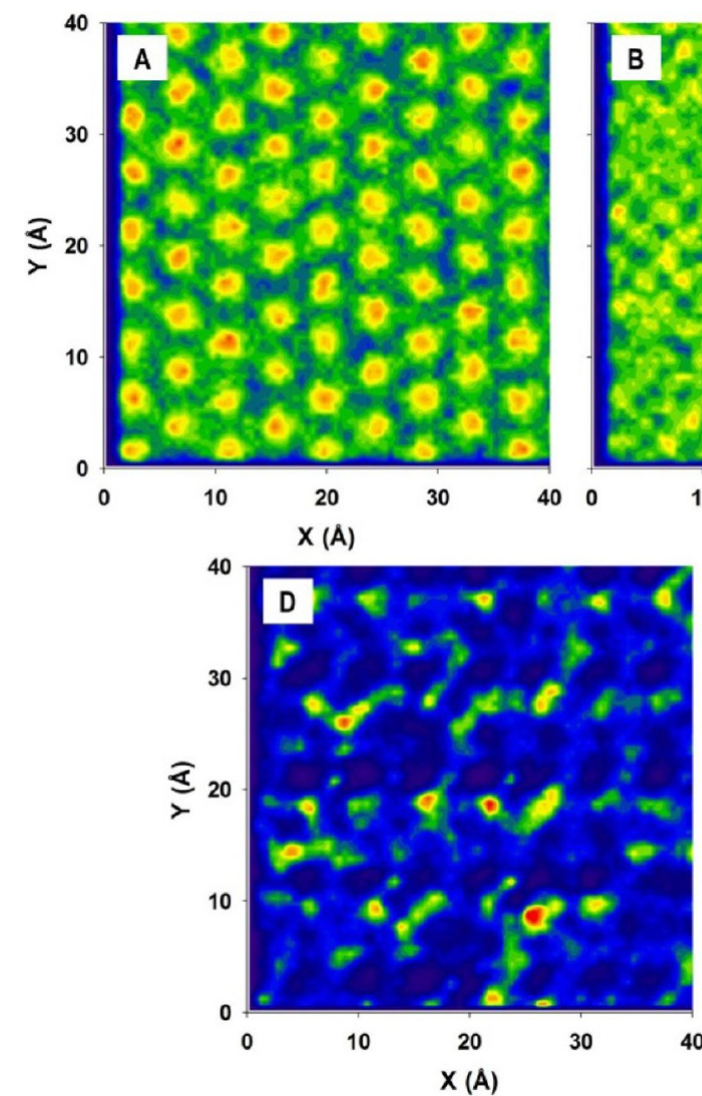
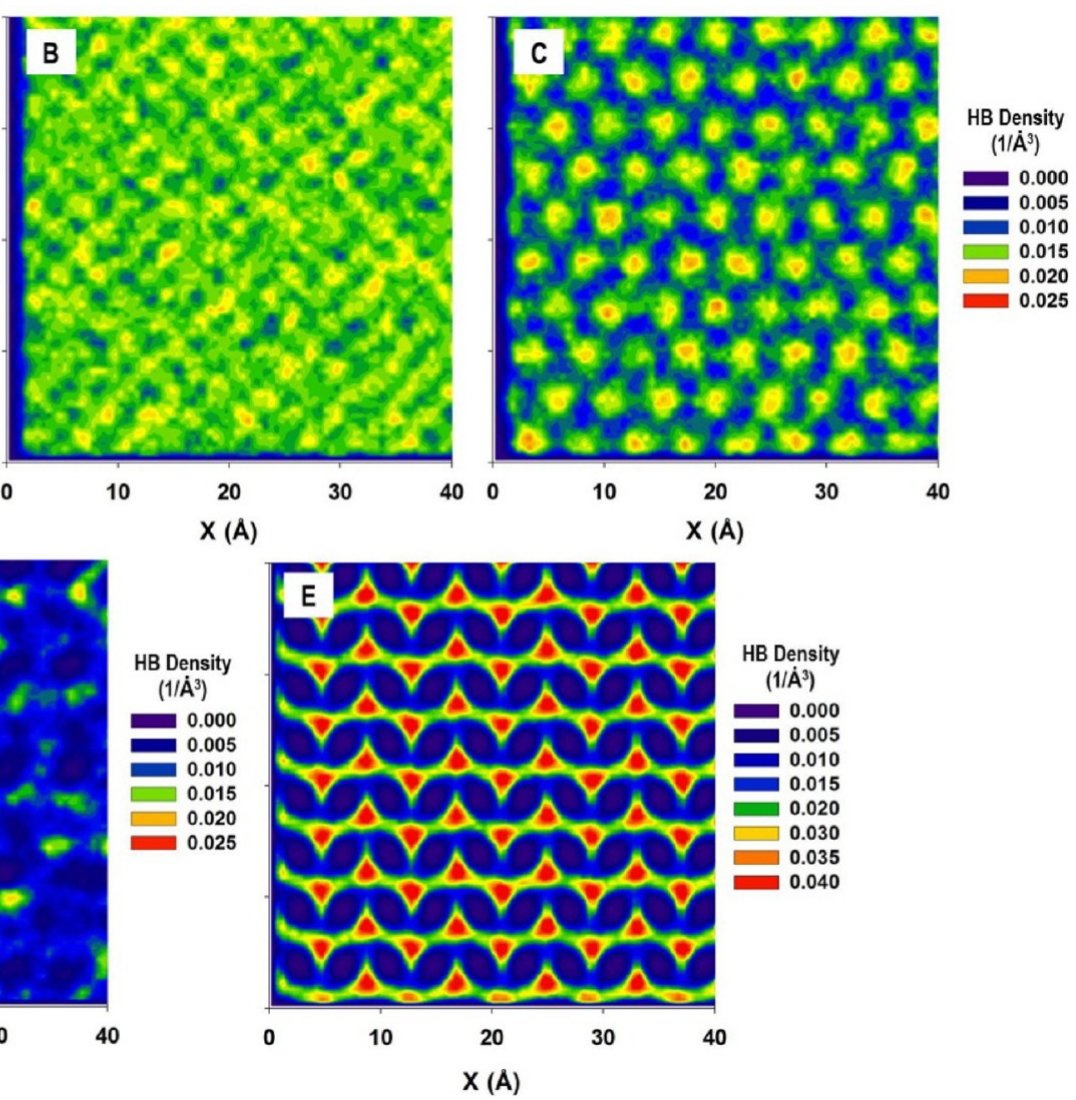

Figure 8. In-plane density distribution of water-water hydrogen bonds within a $40 \times 40 \AA^{2}$ section along the $X-Y$ plane at the center of the hydrated pores. Results are obtained for silica (A), MgO (B), alumina (C), muscovite (D), and calcite (E). Densities are expressed in $1 / \AA^{3}$.
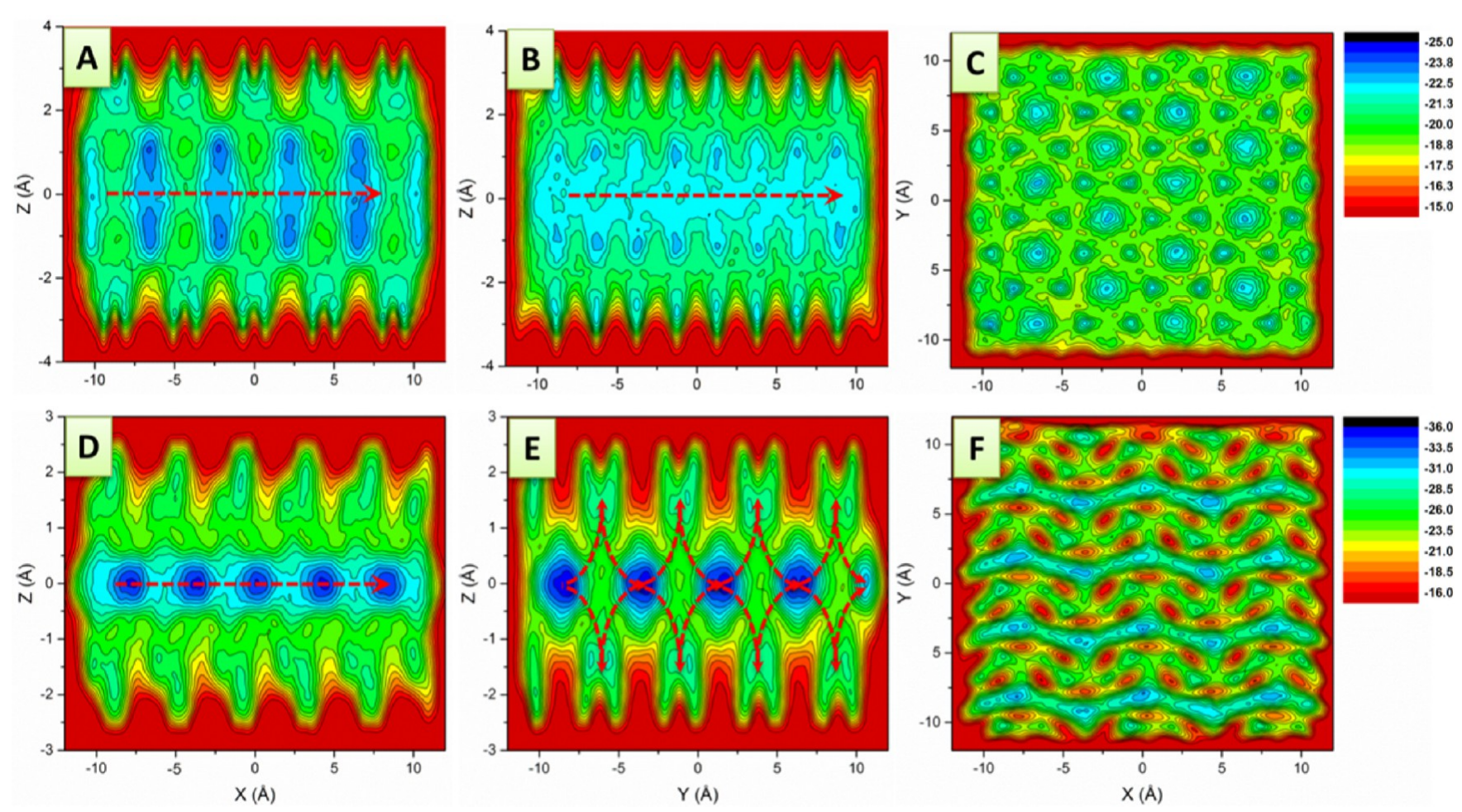

Figure 9. Free energy landscapes projected onto $X-Z(\mathrm{~A}, \mathrm{D}), Y-Z(\mathrm{~B}, \mathrm{E})$, and $X-Y(\mathrm{C}, \mathrm{F})$ planes for silica pore (top panels) and calcite pore (bottom panels) obtained from well-tempered metadynamics simulations. The collective variables chosen are the components of the distance along the three Cartesian coordinates $(X, Y, Z)$ between one methane molecule and the center of the pore. 

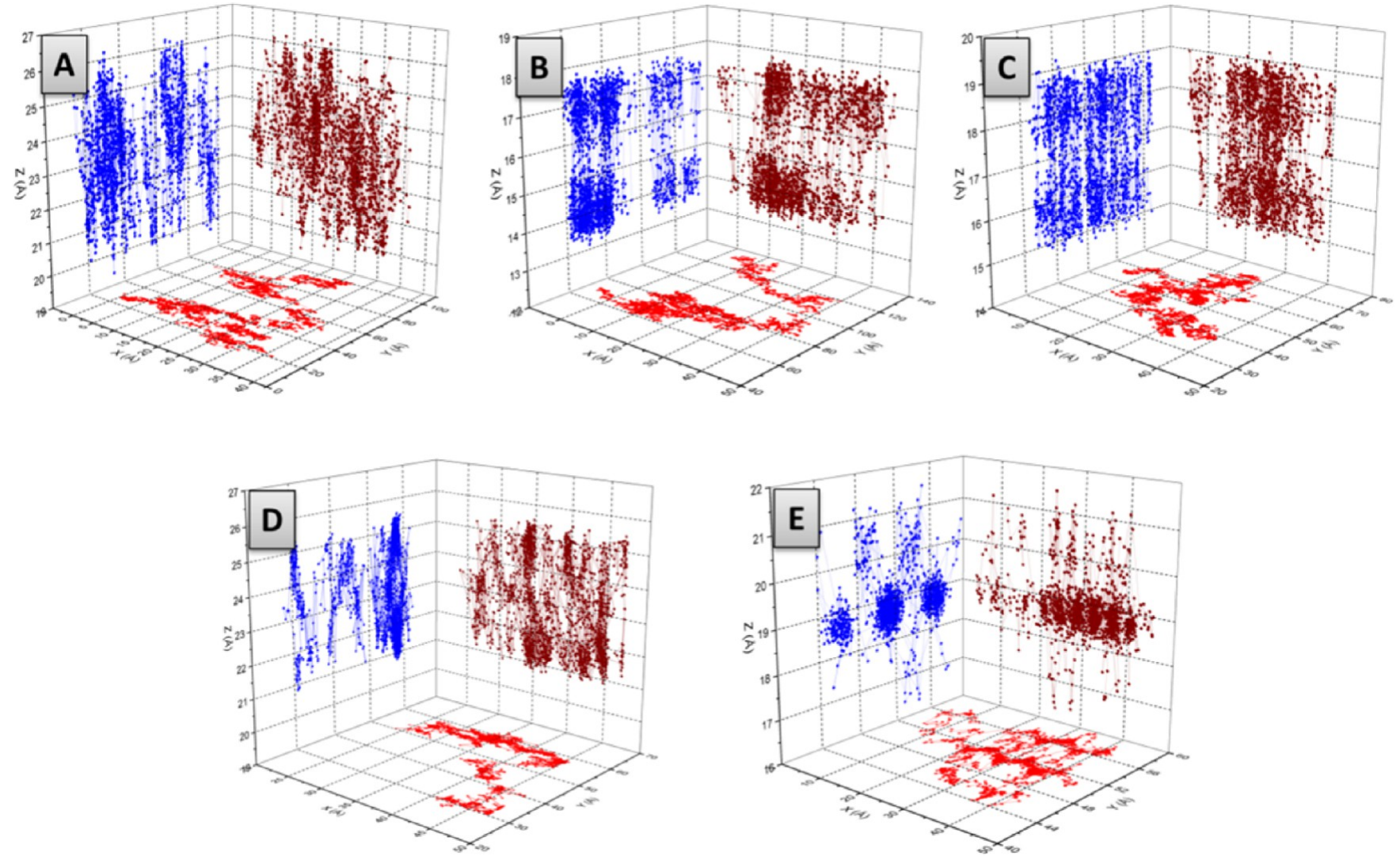

Figure 10. Projection of trajectories of one representative methane molecule in silica (A), MgO (B), alumina (C), muscovite (D), and calcite (E) panel. Red, wine, blue line, and dot represent the trajectories of the methane molecule projected in $X-Y, X-Z$, and $Y-Z$ planes, respectively.

along low free energy regions, which seem to be determined by the specific arrangement of water molecules within the pores.

Analysis of free energy maps suggests favorable pathways for methane diffusion within the hydrated pores. To confirm these results, we monitored the trajectories of representative methane molecules. Visual inspection of the results, presented in Figure 10 , confirms that methane diffuses isotropically within the hydrated silica pore, within which no favorable adsorption of methane occurs along the $Z$ direction other than near the pore center. Within the hydrated $\mathrm{MgO}$, alumina, and muscovite pores, methane molecules preferentially accumulate between the middle of the pore and the pore surfaces (see Figure S4). Even in these pore environments, the trajectories exhibit isotropic transport, even though methane molecules do move from one preferential adsorption site near one surface to one near the opposite surface, and back. The trajectories within the hydrated calcite pore show that methane preferentially accumulates near the middle of the pore (see Figure S4) where it can diffuse along the $X$ direction. To diffuse along the $Y$ direction methane migrates near the pore surfaces and then moves back to the pore center (see the projection onto the $Y-$ $Z$ plane), which is consistent with the free energy maps discussed in Figure 9. Projection of the methane trajectories on the $X-Y$ plane shows that methane molecules move along the $X$ direction following a pattern reminiscent of the HB density distribution shown in panel $\mathrm{E}$ of Figure 6. The distance between two zigzag trajectories in the $Y$ direction is $\sim 5 \AA$, consistent with the one between two adjacent peaks found in the HB density distribution or between two nearby free energy wells in the free energy landscape. This further reinforces our observation that the specific configuration of water network due to synergistic effect of the heterogeneous arrangement of atoms in solid substrate structure and strong interactions between water and solid substrate plays a crucial role in governing the transport of methane guest molecules within hydrated narrow pores. Our results agree qualitatively with those reported by Franco et al., ${ }^{11}$ according to whom the heterogeneous distribution of ions in the calcite substrate was responsible for anisotropic diffusion of fluids in slit-shaped pores. The arrangement of atoms in calcite substrate is illustrated in Figure S14.

\section{CONCLUSIONS}

Atomistic molecular dynamics simulations were employed to quantify the transport of methane through slit-shaped nanopores filled with water. The methane was at low concentration, representing a guest molecule in the hydrated pores, and no chemical potential gradients were imposed on it. The pores were carved out of five solid substrates, chosen because they yield different structures for the confined water molecules. The simulations were conducted at $300 \mathrm{~K}$. The results were analyzed in terms of density profiles, trajectories for the confined methane molecules, self-diffusion coefficients, and free energy maps, obtained implementing metadynamics. It was found that the properties of confined water, in particular its density profiles, strongly affect the preferential adsorption sites for methane molecules. The self-diffusion of confined water, in particular that of the water molecules near the middle of the pore, is correlated with the self-diffusion of methane dissolved in it. However, the self-diffusion coefficient of methane is always lower than that of water. The diffusivity results are in most cases isotropic within the plane parallel to the pore 
surfaces, except when the hydrated calcite pore is considered. In this pore the self-diffusion coefficient for water is slightly greater along the $Y$ direction of the pores (within our representation), whereas the self-diffusion of confined methane is about 3 times larger along the $X$ direction. Solvation free energies, free energy profiles in one direction, density distribution of water molecules in different hydration layers, free energy maps, and preferential diffusion pathways were calculated to explain these differences. The results suggest that many factors are important, including the evolution of solvation free energy distribution within the pores, the structure and dynamics of confined water, and perhaps the heterogeneous water-water hydrogen bond network that strongly affects the preferential diffusion pathways of the guest methane. While experimental verification is needed, the results reveal mechanisms responsible for the anisotropic diffusion of gas molecules in narrow hydrated pores that could be exploited for designing new membranes with better selectivity, or in different separation processes, as well as for assessing and predicting the favorable flow direction of gas migrating in shale formations resulting from hydraulic fracturing operations.

\section{ASSOCIATED CONTENT}

\section{S Supporting Information}

The Supporting Information is available free of charge on the ACS Publications website at DOI: 10.1021/acs.jpcc.7b02713.

Mean-square displacements data for methane and water molecules, density profiles of methane, water and hydrogen bonds along the $Z$ direction, results of potential of mean force profiles calculated using umbrella sampling algorithm as one methane molecule is dragged following one straight line along the $X$ direction of the simulation box, in-plane density distributions of methane and water in the second and third hydration layer, the radial distribution function of water oxygen atoms around methane molecules, residence correlation functions for oxygen atoms in the second and third hydration layers of the hydrated calcite pore, the overlap of free energy landscape projected onto the $X-Y$ plane, and the in-plane density distribution of methane in the hydrated calcite pore (PDF)

\section{AUTHOR INFORMATION}

\section{Corresponding Author}

*E-mail a.striolo@ucl.ac.uk (A.S.).

\section{ORCID}

Alberto Striolo: 0000-0001-6542-8065

\section{Notes}

The authors declare no competing financial interest.

\section{ACKNOWLEDGMENTS}

Generous allocations of computing time were provided by the University College London Research Computing Platforms Support (LEGION), the Oklahoma Supercomputing Center for Education and Research (OSCER), and the National Energy Research Scientific Computing Center (NERSC) at Lawrence Berkeley National Laboratory. NERSC is supported by the DOE Office of Science under Contract DE-AC0205CH11231. A.P. thanks financial support from the Sloan Foundation via the Deep Carbon Observatory and by a Marie Curie Career Initiation Grant awarded to A.S. D.R.C. was supported by the Department of Energy, Office of Basic Energy Sciences, Geosciences program under Grant DE-SC0006878. This research received funding from the European Union's Horizon 2020 research and innovation program under Grant Agreement No. 640979 and from a Marie Curie Career Integration Grant to A.S.

\section{REFERENCES}

(1) Mouallem, C.; de Sousa, W. T.; Cabral, I. E.; Curi, A. Perspectives for Use of Hydraulic Fracturing in Oil and Gas Production. REM, Rev. Esc. Minas 2014, 67, 373-378.

(2) Bahrami, H.; Rezaee, R.; Clennell, B. Water Blocking Damage in Hydraulically Fractured Tight Sand Gas Reservoirs: An Example from Perth Basin, Western Australia. J. Pet. Sci. Eng. 2012, 88-89, 100-106.

(3) Bennion, D. B.; Thomas, F. B. Formation Damage Issues Impacting the Productivity of Low Permeability, Low Initial Water Saturation Gas Producing Formations. J. Energy Resour. Technol. 2005, 127, 240-247.

(4) He, K.; Xu, L.; Gao, Y.; Yin, X.; Neeves, K. B. Evaluation of Surfactant Performance in Fracturing Fluids for Enhanced Well Productivity in Unconventional Reservoirs Using Rock-on-a-Chip Approach. J. Pet. Sci. Eng. 2015, 135, 531-541.

(5) Gardner, P. Microfabricated Nanochannel Implantable Drug Delivery Devices: Trends, Limitations and Possibilities. Expert Opin. Drug Delivery 2006, 3, 479-487.

(6) Ferrati, S.; Fine, D.; You, J.; De Rosa, E.; Hudson, L.; Zabre, E.; Hosali, S.; Zhang, L.; Hickman, C.; Sunder Bansal, S.; et al. Leveraging Nanochannels for Universal, Zero-Order Drug Delivery in Vivo. J. Controlled Release 2013, 172, 1011-1019.

(7) Pérez-Hernández, N.; Luong, T. Q.; Febles, M.; Marco, C.; Limbach, H. H.; Havenith, M.; Pérez, C.; Roux, M. V.; Pérez, R.; Martín, J. D. The Mobility of Water Molecules through Hydrated Pores. J. Phys. Chem. C 2012, 116, 9616-9630.

(8) Zang, J.; Konduri, S.; Nair, S.; Sholl, D. S. Self-Diffusion of Water and Simple Alcohols in Single-Walled Aluminosilicate Nanotubes. ACS Nano 2009, 3, 1548-1556.

(9) Gubbins, K. E.; Long, Y.; Śliwinska-Bartkowiak, M. Thermodynamics of Confined Nano-Phases. J. Chem. Thermodyn. 2014, 74, 169183.

(10) Wang, J.; Kalinichev, A. G.; Kirkpatrick, R. J. Effects of Substrate Structure and Composition on the Structure, Dynamics, and Energetics of Water at Mineral Surfaces: A Molecular Dynamics Modeling Study. Geochim. Cosmochim. Acta 2006, 70, 562-582.

(11) Franco, L. F. M.; Castier, M.; Economou, I. G. Anisotropic Parallel Self-Diffusion Coefficients near the Calcite Surface: A Molecular Dynamics Study. J. Chem. Phys. 2016, 145, 084702.

(12) Phan, A.; Cole, D. R.; Weiß, R. G.; Dzubiella, J.; Striolo, A. Confined Water Determines Transport Properties of Guest Molecules in Narrow Pores. ACS Nano 2016, 10, 7646-7656.

(13) Bollinger, J. A.; Carmer, J.; Jain, A.; Truskett, T. M. Impact of Solvent Granularity and Layering on Tracer Hydrodynamics in Confinement. Soft Matter 2016, 12, 9561-9574.

(14) Phan, A.; Cole, D. R; Striolo, A. Aqueous Methane in SlitShaped Silica Nanopores: High Solubility and Traces of Hydrates. J. Phys. Chem. C 2014, 118, 4860-4868.

(15) Ho, T. A.; Argyris, D.; Papavassiliou, D. V.; Striolo, A.; Lee, L. L.; Cole, D. R. Interfacial Water on Crystalline Silica: A Comparative Molecular Dynamics Simulation Study. Mol. Simul. 2011, 37, 172195.

(16) Argyris, D.; Tummala, N. R.; Striolo, A.; Cole, D. R. Molecular Structure and Dynamics in Thin Water Films at the Silica and Graphite Surfaces. J. Phys. Chem. C 2008, 112, 13587-13599.

(17) Argyris, D.; Ho, T.; Cole, D. R.; Striolo, A. Molecular Dynamics Studies of Interfacial Water at the Alumina Surface. J. Phys. Chem. C 2011, 115, 2038-2046.

(18) Phan, A.; Cole, D. R.; Striolo, A. Preferential Adsorption from Liquid Water-Ethanol Mixtures in Alumina Pores. Langmuir 2014, 30, 8066-8077. 
(19) Causà, M.; Dovesi, R.; Pisani, C.; Roetti, C. AB Initio HartreeFock Study of the MgO(001) Surface. Surf. Sci. 1986, 175, 551-560.

(20) Scamehorn, C. A.; Hess, a. C.; McCarthy, M. I. Correlation Corrected Periodic Hartree-Fock Study of the Interactions between Water and the (001) Magnesium Oxide Surface. J. Chem. Phys. 1993, 99, 2786.

(21) Ho, T. A.; Striolo, A. Water and Methane in Shale Rocks: Flow Pattern Effects on Fluid Transport and Pore Structure. AIChE J. 2015, 61, 2993-2999.

(22) Kerisit, S.; Parker, S. C. Free Energy of Adsorption of Water and Metal Ions on the $\{10 \overline{1} 4\}$ Calcite Surface. J. Am. Chem. Soc. 2004, 126, 10152-10161.

(23) Refson, K.; Wogelius, R. A.; Fraser, D. G.; Payne, M. C.; Lee, M. H.; Milman, V. Water Chemisorption and Reconstruction of the $\mathrm{MgO}$ Surface. Phys. Rev. B: Condens. Matter Mater. Phys. 1995, 52, 1082310826.

(24) Kerisit, S.; Parker, S. C.; Harding, J. H. Atomistic Simulation of the Dissociative Adsorption of Water on Calcite Surfaces. J. Phys. Chem. B 2003, 107, 7676-7682.

(25) Lardge, J. S.; Duffy, D. M.; Gillan, M. J. Investigation of the Interaction of Water with the Calcite (10.4) Surface Using Ab Initio Simulation. J. Phys. Chem. C 2009, 113, 7207-7212.

(26) Cygan, R. T.; Liang, J.-J.; Kalinichev, A. G. Molecular Models of Hydroxide, Oxyhydroxide, and Clay Phases and the Development of a General Force Field. J. Phys. Chem. B 2004, 108, 1255-1266.

(27) Xiao, S.; Edwards, S. A.; Gräter, F. A New Transferable Forcefield for Simulating the Mechanics of $\mathrm{CaCO} 3$ Crystals. J. Phys. Chem. C 2011, 115, 20067-20075.

(28) Martin, M. G.; Siepmann, J. I. Transferable Potentials for Phase Equilibria. 1. United-Atom Description of N -Alkanes. J. Phys. Chem. B 1998, 102, 2569-2577.

(29) Berendsen, H. J. C.; Grigera, J. R.; Straatsma, T. P. The Missing Term in Effective Pair Potentials. J. Phys. Chem. 1987, 91, 6269-6271.

(30) Miyamoto, S.; Kollman, P. A. Settle: An Analytical Version of the SHAKE and RATTLE Algorithm for Rigid Water Models. J. Comput. Chem. 1992, 13, 952-962.

(31) Jorgensen, W. L.; Chandrasekhar, J.; Madura, J. D.; Impey, R. W.; Klein, M. L. Comparison of Simple Potential Functions for Simulating Liquid Water. J. Chem. Phys. 1983, 79, 926.

(32) Jorgensen, W. L.; Maxwell, D. S.; Tirado-Rives, J. Development and Testing of the OPLS All-Atom Force Field on Conformational Energetics and Properties of Organic Liquids. J. Am. Chem. Soc. 1996, $118,11225-11236$.

(33) Essmann, U.; Perera, L.; Berkowitz, M. L.; Darden, T.; Lee, H.; Pedersen, L. G. A Smooth Particle Mesh Ewald Method. J. Chem. Phys. 1995, 103, 8577-8593.

(34) Lorentz, H. A. Ueber Die Anwendung Des Satzes Vom Virial in Der Kinetischen Theorie Der Gase. Ann. Phys. 1881, 248, 127-136.

(35) Berthelot, D. Sur Le Mélange Des Gaz. Compt. Rendus 1898, 126, 1703-1706.

(36) Van Der Spoel, D.; Lindahl, E.; Hess, B.; Groenhof, G.; Mark, A. E.; Berendsen, H. J. C. GROMACS: Fast, Flexible, and Free. J. Comput. Chem. 2005, 26, 1701-1718.

(37) Abraham, M. J.; Murtola, T.; Schulz, R.; Páll, S.; Smith, J. C.; Hess, B.; Lindahl, E. GROMACS: High Performance Molecular Simulations through Multi-Level Parallelism from Laptops to Supercomputers. SoftwareX 2015, 1-2, 19-25.

(38) Hoover, W. G. Canonical Dynamics: Equilibrium Phase-Space Distributions. Phys. Rev. A: At., Mol., Opt. Phys. 1985, 31, 1695-1697.

(39) Nosé, S. A Molecular Dynamics Method for Simulations in the Canonical Ensemble. Mol. Phys. 1984, 52, 255-268.

(40) Hockney, R. W.; Goel, S. P.; Eastwood, J. W. Quiet HighResolution Computer Models of a Plasma. J. Comput. Phys. 1974, 14, $148-158$.

(41) Barducci, A.; Bussi, G.; Parrinello, M. Well-Tempered Metadynamics: A Smoothly Converging and Tunable Free-Energy Method. Phys. Rev. Lett. 2008, 100, 020603.

(42) Bonomi, M.; Branduardi, D.; Bussi, G.; Camilloni, C.; Provasi, D.; Raiteri, P.; Donadio, D.; Marinelli, F.; Pietrucci, F.; Broglia, R. A.; et al. PLUMED: A Portable Plugin for Free-Energy Calculations with Molecular Dynamics. Comput. Phys. Commun. 2009, 180, 1961-1972.

(43) Gubbins, K. E.; Liu, Y.-C.; Moore, J. D.; Palmer, J. C. The Role of Molecular Modeling in Confined Systems: Impact and Prospects. Phys. Chem. Chem. Phys. 2011, 13, 58-85.

(44) Ritos, K.; Mattia, D.; Calabrò, F.; Reese, J. M. Flow Enhancement in Nanotubes of Different Materials and Lengths. J. Chem. Phys. 2014, 140, 014702.

(45) Limmer, D. T.; Willard, A. P.; Madden, P.; Chandler, D. Hydration of Metal Surfaces Can Be Dynamically Heterogeneous and Hydrophobic. Proc. Natl. Acad. Sci. U. S. A. 2013, 110, 4200-4205.

(46) Hummer, G.; Garde, S.; García, a E.; Pohorille, A.; Pratt, L. R. An Information Theory Model of Hydrophobic Interactions. Proc. Natl. Acad. Sci. U. S. A. 1996, 93, 8951-8955.

(47) Ringnér, M. What Is Principal Component Analysis? Nat. Biotechnol. 2008, 26, 303-304.

(48) Witherspoon, P. A.; Saraf, D. N. Diffusion of Methane, Ethane, Propane, and N-Butane in Water from 25 to $43^{\circ}$. J. Phys. Chem. 1965, 69, 3752-3755.

(49) Shvab, I.; Sadus, R. J. Thermodynamic Properties and Diffusion of Water + Methane Binary Mixtures. J. Chem. Phys. 2014, 140, 104505 .

(50) Gillen, K. T.; Douglass, D. C.; Hoch, M. J. R. Self-Diffusion in Liquid Water to $-31^{\circ}$ C. J. Chem. Phys. 1972, 57, 5117.

(51) Smith, D. E.; Dang, L. X. Computer Simulations of $\mathrm{NaCl}$ Association in Polarizable Water. J. Chem. Phys. 1994, 100, 3757.

(52) Mark, P.; Nilsson, L. Structure and Dynamics of the TIP3P, SPC, and SPC/E Water Models at 298 K. J. Phys. Chem. A 2001, 105, 9954-9960.

(53) Liu, Y.; Wang, Q.; Lu, L. Transport Properties and Distribution of Water Molecules Confined in Hydrophobic Nanopores and Nanoslits. Langmuir 2004, 20, 6921-6926.

(54) Chiavazzo, E.; Fasano, M.; Asinari, P.; Decuzzi, P. Scaling Behaviour for the Water Transport in Nanoconfined Geometries. Nat. Commun. 2014, 5, 4565.

(55) Bourg, I. C.; Steefel, C. I. Molecular Dynamics Simulations of Water Structure and Diffusion in Silica Nanopores. J. Phys. Chem. C 2012, 116, 11556-11564.

(56) Mitra, S.; Mukhopadhyay, R.; Tsukushi, I.; Ikeda, S. Dynamics of Water in Confined Space (Porous Alumina): QENS Study. J. Phys.: Condens. Matter 2001, 13, 8455.

(57) Kästner, J. Umbrella Sampling. Wiley Interdiscip. Rev. Comput. Mol. Sci. 2011, 1, 932-942.

(58) Jamadagni, S. N.; Godawat, R.; Garde, S. Hydrophobicity of Proteins and Interfaces: Insights from Density Fluctuations. Annu. Rev. Chem. Biomol. Eng. 2011, 2, 147-171.

(59) Fenter, P.; Kerisit, S.; Raiteri, P.; Gale, J. D. Is the Calcite-Water Interface Understood? Direct Comparisons of Molecular Dynamics Simulations with Specular X-Ray Reflectivity Data. J. Phys. Chem. C 2013, 117, 5028-5042.

(60) Argyris, D.; Ashby, P. D.; Striolo, A. Structure and Orientation of Interfacial Water Determine Atomic Force Microscopy Results: Insights from Molecular Dynamics Simulations. ACS Nano 2011, 5, 2215-2223.

(61) Phan, A.; Ho, T. A.; Cole, D. R.; Striolo, A. Molecular Structure and Dynamics in Thin Water Films at Metal Oxide Surfaces: Magnesium, Aluminum, and Silicon Oxide Surfaces. J. Phys. Chem. C 2012, 116, 15962-15973.

(62) Skipper, N. T. Computer Simulation of Methane-water Solutions. Evidence for a Temperature-Dependent Hydrophobic Attraction. Chem. Phys. Lett. 1993, 207, 424-429.

(63) Asthagiri, D.; Merchant, S.; Pratt, L. R. Role of Attractive Methane-Water Interactions in the Potential of Mean Force between Methane Molecules in Water. J. Chem. Phys. 2008, 128, 244512.

(64) Matubayasi, N.; Reed, L. H.; Levy, R. M. Thermodynamics Of The Hydration Shell 0.1. Excess Energy Of A Hydrophobic Solute. J. Phys. Chem. 1994, 98, 10640-10649. 